\title{
Exposição ocupacional ao ruído e hipertensão arterial*
}

\author{
Occupational noise exposure and hypertension
}

\author{
Vilma S. Santana e João Luiz Barberino \\ Instituto de Saude Coletiva da Universidade Federal da Bahia. Salvador - Brasil (V.S.S.), Departamento de \\ Medicina Preventiva da Faculdade de Medicina da Universidade Federal da Bahia. Salvador - Brasil (J.L.B.)
}

\begin{abstract}
A hipótese de que a exposição ao ruído ocupacional estava positivamente associada à hipertensăo arterial foi avaliada em um estudo transverșal, reałizado com um grupo de 276 pacientes, admitidos em um ambulatório de saúde do trabalhador do Sistema Único de Saúde, atendidos nos primeiros seis meses de 1992. A exposiçăo ao ruído teve duas medidas: história referida de exposição ocupacional ao ruído e o diagnóstico de disacusia ocupacional. A hipertensāo arterial foi definida de acordo com os critérios da OMS, incluindo-se também a referéncia a tratamento anti-hipertensivo. Dados obtidos através da análise estratificada $\theta$ da modelagem logística nāo-condicional revelam que a hipótese näo foi confirmada: nâo se encontrou diferenças entre a pressão sistólica ou diastólica ou entre as proporçठ̄es de hipertensāo entre indivíduos expostos ou năo expostos. Todavia, verificou-se aumento estatisticamente significante $(a \mid f a=0,05)$ da medida de efeito quando o nivel de educaçäo era baixo (até o primeiro grau completo), o que parece indicar maior intensidade ou duração da exposiçāo entre os trabalhadores desse grupo. Isto pode ser outra evidência da desigualdade social subjacente à distribuiçăo da exposiçăo entre trabalhadores no ambiente de trabalho, o que deverá ser focalizado. com mais profundidade, em estudos futuros.
\end{abstract}

Hipertensâo, epidemiologia. Ruido ocupacional, efeito adverso. Perda auditiva provocada por ruido, epidemiologia.

\section{Introdução}

Resultados de estudos experimentais conduzidos com animais apontam para uma elevação de níveis tensionais com exposição ao ruído:. Em seres humanos, são vários os estudos que avaliaram a hipótese de que trabalhadores expostos ao ruído nos locais de trabalho poderiam apresentar um excesso de casos de hipertensão arterial. Takala e col..$^{22}$, por exemplo, compararam medidas de pressão arterial em um grupo de trabalhadores com perda auditiva e outro sem perda auditiva, verificando não haver diferenças entre as médias tensionais, seja sistólica ou diastólica, embora assinalassem que a média de idade do grupo com perda auditiva era 10 anos maior do que a do grupo sem perda auditiva. Consistentemente, no estudo desenvolvido por Malchaire e Mullier ${ }^{i 6}$, concluiram-se que não havia indícios de uma associação positiva entre trabalho em indústrias que se caracterizavam por ruído excessivo (92 a 100 decibéis dBA) e hipertensão arterial, embora os dados apresentados nāo traduzam evidências epidemiológicas que permitam essas afirmaçōes.

Em uma direção oposta, situam-se os dados da pesquisa desenvolvida por Jonsson e Hanson que encontraram níveis tensionais sistólico e diastólico mais elevados entre trabalhadores industriais com disacusia ocupacional, quando comparados com as medidas obtidas entre outros da mesma idade que não apresentavam disfunção auditiva. Similarmente, Verbeeck e col. ${ }^{2.9}$ estudaram a pressão arterial sistólica e diastólica de 297 trabalhadores de indústrias que apresentavam níveis de ruído no ambiente de trabalho acima de $80 \mathrm{dBA}$, encontrando uma tendência de elevação de ambos os dados com o aumento da duração da exposição

"Trabalho apresentado no N Congresso Brasileiro de Saxde Coletiva. Recife. PE, 1994.

Separatas/Reprints: VilmaS. Santana - Instituto de Sáide Coletiva da Universidade Federal da Bahia. Rua Padre Feijó, $29.4^{\circ}$ andar-40110-117 - Salvador, BA - Brasil. Fax: (071) 237.5856 E-mail: Vilma@ UFBA.BR

Recebido em 27.12.1994. Aprovado em 8.8.1995. 
(Coeficiente de Correlação de Pearson de 0,29 e 0,31 , respectivamente, ambos estatisticamente significantes para um alfa $=0,05$ ) que se mantinha mesmo após o ajuste por idade.

Parvizpoor ${ }^{19}$ comparou a frequiência de hipertensão arterial de um grupo de 821 tecelōes empregados em fábricas com níveis de exposiçāo ao ruído em torno de $96 \mathrm{dBA}$, com os valores tensionais de 412 trabalhadores de condiçôes socioeconômicas semelhantes, que trabalhavam em indústrias que não se caracterizavam por ruído elevado. Estimou-se uma razão de prevalência de hipertensão de 3,5:1 (expostos versus não expotos) e para casos "borderline" $2,7: 1$, enquanto que a idade de início do efeito entre os expostos foi, em média, 10 anos mais baixa do que entre os não expostos. Mais recentemente, Zhao e col.24, utilizando análise logística năo condicional, verìficaram um incremento da razâo dos produtos cruzados ("odds ratio") de $100 \%$ para uma variação de $30 \mathrm{dBA}$ no nível de exposição, independentemente de fatores considerados "confundíveis" como a história familiar de hipertensão, ingesta de sal e idade. Observou-se, ainda, que a associação entre a prevalência de hipertensão arterial e exposição ao ruído evidenciava tendência dose-resposta para as medidas brutas, o que persistia mesmo após o ajuste pelas variáveis confundidoras.

O substrato físiopatológico da hipótese de que a exposição ao ruído excessivo determina hipertensão arterial é de que reações do sistema circulatório ao estresse, como o aumento das catecolaminas na corrente sangüínea, a vasoconstrição periférica e a elevação da frequiência cardiáca estão associados com o aumento da pressão arterial temporária ${ }^{2}$. Esta elevação temporária poderia ser um marcador de indíviduos susceptíveis ao desenvolvimento de hipertensão arterial. Ressaitase, portanto, que a trilha de causalidade ("causal pathway") plausível para a hipótese em consideração seja a bioquímica relacionada com os mecanismos do estresse.

A hipertensão arterial é enfermidade de aita prevalência no Brasil ${ }^{j .13 .14}$ e a exposição ao ruído excessivo em ambientes ocupacionais tem sido citada como o fator de risco industrial mais comum. A disacusia ocupacional, por sua vez, predomina na demanda atendida em serviços especializados em medicina do trabalho, no Brasi1 ${ }^{20}$. Todavia, é bastante limitado, o conhecimento do papel da exposição ao rú́do excessivo na produção de hipertensão arterial. Muitos dos estudos são de natureza exploratória, freqüentemente con- duzidos com dados secundários, numa perspectiva descritiva, apresentando apenas comparaçōes de frequiências entre grupos expostos e não expostos. Não há menção a investigação e tratamento analítico apropriados de modificadores de efeito ou confundidores que permitiriam um conhecimento mais conclusivo sobre essa questâo.

O Centro de Estudos em Saúde do Trabalhador, do Sistema Único de Saúde (SUS) da Bahia, através da sua Unidade Ambulatorial, atende trabalhadores encaminhados para avaliação clínica ocupacional. Um dos objetivos dessa unidade é a construçâo de uma base de dados para a realização de estudos epidemiológicos, mediante a documentação detalhada de informações sobre história de exposições ocupacionais e demais dados de interesse clínico. No presente estudo pretende-se verificar a direção e magnitude da associação entre a exposição ao ruído no ambiente do trabalho e a hipertensão arterial. A existência de informações sobre algumas características dos indivíduos permitirá, ainda que exploratoriamente, avaliar o papel de variáveis de confundimento ou modificadoras de efeito, aspectos não contemplados em outros estudos sobre este tema.

\section{Metodologia}

Conduziu-se um estudo de corte transversal com dados existentes em 507 prontuários médicos da Unidade Ambulatorial do Centro de Saúde do Trabalhador, SUS/Bahia, com toda a clientela admitida entre 1 de janeiro a 31 de dezembro de 1992. Descartaram-se 15 prontuários $(0,9 \%)$ que não apresentavam informações. Dos 492,216 não apresentaram informações sobre as variáveis relacionadas com a hipótese principal, história de exposição ocupacional ao ruído e hipertensão arterial, o que implicou sua exclusão da análise. Os restantes 276 ficaram distribuídos em dois grupos de análise: os que dispunham de dados para o estudo da história de exposição ao ruído ocupacional e hipertensão (276) e os com informações para a variávej disacusia ocupacional e hipertensão (231).

Para a coleta de dados foram treinados 50 alunos do curso de medicina da Universidade Federal da Bahia. Foram selecionadas as informaçōes a serem analisadas, que eram classificadas, codificadas e registradas em um formulário próprio. Concluída a coleta, os dados eran digitados e submetidos a avaliação de concordância com os dados dos formulários. Após as correções necessárias, os dados eram analisados utilizando- 
se "Statistical Package for Statisticians/SAS", versão 6.04 .

A variável independente, exposição ocupacional ao ruído, teve duas medidas baseadas nas informações existentes nos prontuários. Uma delas foi a história de exposição ao ruído em local de trabalho colhida por assistentes sociais, que compunha a ficha de história de exposições ocupacionais, que era complementada por uma ficha especial para o ruído, caso necessário. A outra medida da variável independente principal foi o diagnóstico de disacusia ocupacional, quando nāo era resultante de trauma sonoro súbito, definido com o laudo de nexo causal elaborado para fins jurídico-legais, por médicos especialistas. Os pacientes que não dispunham desse laudo ou que obtiveram outros diagnósticos, com ou sem nexo ocupacional, foram incluídos na categoria de "não presença đe disacusia ocupacional". Ambas as variáveis foram analisadas dicotomicamente ( $1=$ exposto; $0=$ não exposto).

A variável de efeito, hipertensão arterial, foi definida com base nas medidas tensionais tomadas na admissão. As medidas eram feitas pelos médi$\cos$ da equipe, com os pacientes sentados empregando-se esfigmomanômetro braquial. Definiramse duas variáveis dependentes como medidas do efeito: uma contínua, os níveis tensionais sistólico e diastólico, individuais, e uma dicotômica. o diagnóstico de hipertensão arterial definido segundo os critérios propostos pela Organização Mundial da Saúde ${ }^{18}$, incluindo-se a história de tratamento atual para a hipertensão arterial. Especificamente, foram considerados como hipertensos os indíviduos que obtiveram pelo menos um dos seguintes dados: nível tensional sistólico acima de $160 \mathrm{~mm}$ de mercúrio $(\mathrm{mmHg}$ ), diastólico acima de $95 \mathrm{~mm} \mathrm{Hg}$, e referência de tratamento atual. Não havia dados sobre história de níveis tensionais conhecidos ou hipertensão arterial referida embora houvesse registro de uso de tratamento anti-hipertensivo.

A hipótese operacional do estudo foi analisada através da comparação entre a freqüência de hipertensão arterial entre os indivíduos que mencionaram história de exposição ao ruído em local de trabalho, com aquela estimada entre os pacientes que não registraram este tipo de exposição ao ruído. Complementarmente, foram também comparadas as frequiências de hipertensão entre os indivíduos que obtiveram o diagnóstico de disacusia ocupacional através do laudo de nexo causal, com as proporçōes obtidas para o grupo de trabalhadores com outros diagnósticos. Razões das proporçōes foram estimadas para a avaliação da magnitude e direção da associação, enquanto que a inferência estatística baseou-se em intervalos de confiança estimados pelo método das Séries de Taylor" e testes do Qui quadrado para um alfa de 0,05 . Para as comparaçōes das médias tensionais utilizou-se o teste $\mathrm{t}$ de "Student", com distribuiçōes checadas para a normalidade empregando-se o gráfico da distribuição estandardizada e o teste de Kolmogorov-Smirnof:"

Modificação de efeito foi avaliada para as covariáveis ocupação, educação. idade e sexo, considerando-se afastamentos de modelos multiplicativos. Para aquelas variáveis que eram intrinsecamente vinculadas à exposição ou a algum dos efeitos, do ponto de vista teórico-conceitual, como por exemplo a idade, que presumivelmente indica duraçāo da exposição ou para aquelas nas quais se identificou empiricamente a presença de modificaçāo de efeito, o papel de variável de confundimento não foi avaliado. Considerou-se variável modificadora de efeito aquelas que apresentavam evidência de "interação estatística" seja na análise estratificada ou multivariada ${ }^{6.11} . \mathrm{Na}$ análise tabular, considerou-se variável confundidora todas aquelas que apresentaram associação com a hipertensão arterial entre os não expostos ao ruído, e simultaneamente, com a exposição ao ruído no grupo de não hipertensos".

A análise multivariada foi empregada no estudo de apenas uma das variáveis de efeito, a hipertensāo e não hipertensão, utilizando-se modelo logístico não-condicional porque o efeito era dicotômico e o desenho do estudo era não pareado. A medida contínua do nível tensional foi julgada como tendo pequeno valor interpretativo epidemiológico neste contexto, no qual a população se constituía de pacientes de um serviço de referência onde o uso de tratamento era provável, caso fossem hipertensos. Definiu-se a codificação das variáveis de modo a permitir a sua linearidade. A modelagem foi conduzida adotando-se o procedimento chamado de "backward", tanto para a estimativa da modificação de efeito como para o confundimento. Assim, numa primeira etapa, todos os termos, simples e os correspondentes termos-produto eram incluídos na equação. Em seguida, avaliava-se modificação de efeito sob pressupostos multiplicativos, estimandose a razāo de verossimilhança (teste da razão do -2 In da verossimilhança) correspondente à cada uma das covariáveis. Considerando-se como modificador de efeito, aquelas covariáveis que alteravam o ajustamento do modelo em níveis estatisticamente significantes. A avaliação de confundimento baseou-se na verificação de alteraçōes nas estimativas dos "odds ratio" (e $\beta$ ) correspondentes a associ- 
ação entre exposiçāo ao ruído e hipertensāo arterial, bem como alteraçōes da amplitude do respectivo intervalo de confrança, induzido pela retirada do termo da equaçãó.

$\mathrm{O}$ grupo de indivíduos com dados não obtidos para as variáveis correspondentes ao efeito principal, exposição ao ruído e hipertensão arterial foi submetido a uma avaliação descritiva da sua composição para identificaçāo de suas características e de como estas poderiam se constituir em possíveis fontes de viezes a serem avaliadas durante a análise.

\section{Resultados}

Na Tabela 1 são apresentadas as distribuições de idade, sexo, educação e ocupação para o grupo que tinha informações para a história de exposição ao ruído $\left(\mathrm{n}^{\circ}=276\right)$ e para aquele com dados não obtidos $\left(n^{Q}=216\right)$. Baseando-se nos resultados dos testes Qui quadrado, não foram encontradas diferenças estatisticamente significantes para nenhuma das covariáveis (alfa $=0,05$ ) em suas distribuiçôes nesses grupos.

Para avaliar a concordância entre as medidas das variáveis, comparou-se a história de exposição ao ruído ocupacional com a disacusia ocupacional diagnosticada, cujos resultados estão dispostos na Tabela 2. Observou-se que dos 379 indivíduos para os quais havia dados para essas variáveis, apenas um indivíduo obteve o diagnóstico de disacusia ocupacional quando não havia o registro no prontuário de histónia de exposição ocupacional ao ruído. Nesse caso, considerando-se que a medida proveniente da avaliação clínico-epidemiológica do nexo ocupacional é mais acurada do que

Tabela 1 - Características dos individuos de acordo com as informaçōes disponiveis para as variáveis de exposiçāo ao ruido ocupacional e hipertensăo arterial.

\begin{tabular}{crrrrrrr}
\hline & \multicolumn{3}{c}{ Com Inf. } & \multicolumn{3}{c}{ Sem Inf. } & \multicolumn{2}{c}{ Total } \\
\cline { 2 - 7 } Covariaveis & $\mathrm{N}=276$ & $(\%)$ & $\mathrm{N}=216$ & $(\%)$ & $\mathrm{N}=492$ & $(\%)$ \\
\hline Sexo & & & & & & \\
Masc. & 243 & 88,0 & 183 & 85,0 & 426 & 86,6 \\
Fem. & 33 & 12,0 & 33 & 15,0 & 66 & 13,4 \\
Idade & & & & & & \\
19-35 & 81 & 29,4 & 78 & 36,1 & 159 & 32,3 \\
36-45 & 86 & 31,2 & 65 & 30,1 & 151 & 30,0 \\
46-64 & 109 & 39,5 & 73 & 33,8 & 182 & 37,0 \\
Educaçāo & & & & & & \\
Alta & 164 & 59,4 & 114 & 52,8 & 278 & 56,5 \\
Baixa & 112 & 40,6 & 102 & 47,2 & 214 & 43,5 \\
Qualificaçăo & & & & & & \\
Profissional & & & & & & \\
Alta & 179 & 64,9 & 118 & 54,6 & 297 & 60,4 \\
Baixa & 97 & 35,1 & 98 & 45,4 & 195 & 39,6 \\
\hline
\end{tabular}

mero registro da exposição no prontuário, imputou-se como positiva a resposta corresponde à história de exposição ao ruído ocupacional.

$\mathrm{Na}$ Tabela 3 são apresentadas as distribuiçōes das covariáveis de acordo com a história de exposição ocupacional ao rú́do. Verificou-se que entre aqueles com história de exposiçāo ao ruído

Tabela 2 - Comparação entre as medidas de exposição ao ruido ocupacional utilizadas.

\begin{tabular}{lrcc}
\hline Disacusia & História referida de exposição ao rúdo \\
\cline { 3 - 4 } Ocupacional & Com & Sem & Total \\
\cline { 3 - 4 } Com & 174 & $0^{*}$ & 174 \\
Sem & 58 & 147 & 205 \\
\hline Total & 232 & 147 & 379 \\
\hline
\end{tabular}

*Foi corrigida a informação sobre um indivíduo que referiu história negativa para exposiçăo ao ruído ocupacional, mas que obteve diagnóstico de disacusia ocupacional.

no local de trabalho existia predominância de indivíduos do sexo masculino ( $\mathrm{X} 2=58,87,1 \mathrm{gl}$, $\mathrm{p}<0,001)$ e discreta concentraçāo de pessoas mais jovens entre os nāo expostos, que atinge apenas um nível "bordeline" de significância estatística (X2=5,693, 2gl, p=0,058). Não houve diferenças significantes nas composições desses grupos para as variáveis educação ou nível de qualificação profissional. Este mesmo procedimento foi conduzido com a variável disacusia ocupacional (Tabela 4), evidenciando-se padrões de distribuição semelhantes àqueles obtidos com a história de exposição ocupacional ao ruído.

As distribuiçōes dos valores tensionais sistólico e diastólico foram "normais" nāo se encontrando medidas tensionais extremas. Analisando-se as médias das tensões sistólica para os grupos exposto e não exposto, de acordo com a variável história de exposição ocupacional ao ruído, estimou-se $130,0 \mathrm{mmHg} \quad\left(\mathrm{DP}^{*}=24,0\right)$ e $126,0 \mathrm{mmHg}$ $(\mathrm{DP}=18,5)$, respectivamente, diferença não estatisticamente significante (Tabela 5). Para a variável disacusia ocupacional os dados correspondentes são $128,9 \mathrm{mmHg}(\mathrm{DP}=20,6)$ e $124,8 \mathrm{mmHg}$ $(\mathrm{DP}=21,0)$, cuja diferença também não é estatisticamente significante.

Analogamente, as medidas de tensāo diastólica mostraram diferenças nāo estatisticamente significantes para os grupos exposto e não exposto, em referência à história de exposição ao rúdo, $84,3 \mathrm{mmHg}(\mathrm{DP}=10,9)$ e $83,1 \mathrm{mmHg}(\mathrm{DP}=13,2)$, enquanto que para aqueles com disacusia ocupa-

* Desvio -padrão. 
cional os resultados foram $84,1 \mathrm{mmHg}(\mathrm{DP}=10,9) \mathrm{e}$ $83,1 \mathrm{mmHg}(\mathrm{DP}=13,2)$, respectivamente (Tabela 5).

A proporção de hipertensão arterial entre os indivíduos com história de exposição ao ruído ocupacional foi menor $(20,3 \%)$ do que entre aqueles com história negativa $(23,4 \%)$, conforme os dados apresentados na Tabela 6. Correspondentemente, a hipertensão arterial foi verificada em $20,7 \%$ dos indivíduos que não obtiveram diag-

Tabela 3 - Características da população do estudo, de acordo com a história de exposiçảo ao ruido.

\begin{tabular}{|c|c|c|c|c|c|c|}
\hline \multirow[b]{3}{*}{ Covariáveis } & \multicolumn{6}{|c|}{ História referida de exposiçăo ao ruído } \\
\hline & \multicolumn{2}{|c|}{ Com } & \multicolumn{2}{|c|}{ Sem } & \multicolumn{2}{|c|}{ Total } \\
\hline & $N=212$ & $\%$ & $N=64$ & $\%$ & $N=276$ & $\%$ \\
\hline Sexo & & & & & & \\
\hline $\begin{array}{l}\text { Masc. } \\
\text { Fem. } \\
\text { Idade }\end{array}$ & $\begin{array}{r}203 \\
9\end{array}$ & $\begin{array}{r}95,8 \\
4,3\end{array}$ & $\begin{array}{l}40 \\
24\end{array}$ & $\begin{array}{l}62,5 \\
37,5\end{array}$ & $\begin{array}{r}243 \\
33\end{array}$ & $\begin{array}{l}88,0 \\
12,0\end{array}$ \\
\hline $\begin{array}{l}19-30 \\
31-40 \\
41-64\end{array}$ & $\begin{array}{l}58 \\
64 \\
90\end{array}$ & $\begin{array}{l}27,4 \\
30,2 \\
42,5\end{array}$ & $\begin{array}{l}23 \\
22 \\
19\end{array}$ & $\begin{array}{l}36,0 \\
34,4 \\
29,7\end{array}$ & $\begin{array}{r}81 \\
86 \\
109\end{array}$ & $\begin{array}{l}29,4 \\
31,2 \\
39,5\end{array}$ \\
\hline Educaçäo & & & & & & \\
\hline $\begin{array}{l}\text { Alta } \\
\text { Baixa }\end{array}$ & $\begin{array}{r}86 \\
126\end{array}$ & $\begin{array}{l}40,6 \\
59,4\end{array}$ & $\begin{array}{l}26 \\
38\end{array}$ & $\begin{array}{l}40,6 \\
59,4\end{array}$ & $\begin{array}{l}112 \\
164\end{array}$ & $\begin{array}{l}40,6 \\
59,4\end{array}$ \\
\hline $\begin{array}{l}\text { Qualificaçăo } \\
\text { Profissional }\end{array}$ & & & & & & \\
\hline Alta & 138 & 65,1 & 41 & 64,1 & 179 & 64,9 \\
\hline Baixa & 74 & 34,9 & 23 & 35,9 & 97 & 35,2 \\
\hline
\end{tabular}

Educaçāo (alta = maior do que o $1^{\mathrm{g}} \mathrm{grau}$; baixa = igual ou menor do que o $1^{9} \mathrm{grau}$ )

Profissảo (alta $=$ profissōes que requerem nivel de educaçăo superior, funçōes técnicas qualificadas; baixa = funçōes de média ou baixa qualificaçāo, auxiliares ou atendentes)

Tabela 4 - Características da populaçāo do estudo, de acordo com a disacusia ocupacional.

\begin{tabular}{|c|c|c|c|c|c|c|}
\hline \multirow[b]{3}{*}{ Covariáveis } & \multicolumn{6}{|c|}{ Disacusia ocupacional } \\
\hline & \multicolumn{2}{|c|}{ Com } & \multicolumn{2}{|l|}{ Sem } & \multicolumn{2}{|l|}{ Total } \\
\hline & $N=121$ & $\%$ & $N=110$ & $\%$ & $N=231$ & $\%$ \\
\hline Sexo & & & & & & \\
\hline $\begin{array}{l}\text { Masc. } \\
\text { Fem. } \\
\text { ldade }\end{array}$ & $\begin{array}{c}117 \\
4\end{array}$ & $\begin{array}{c}96,6 \\
3,4\end{array}$ & $\begin{array}{l}80 \\
30\end{array}$ & $\begin{array}{l}72,7 \\
27,3\end{array}$ & $\begin{array}{c}197 \\
34\end{array}$ & $\begin{array}{l}85,3 \\
14,7\end{array}$ \\
\hline $\begin{array}{l}19-30 \\
31-40 \\
41-64\end{array}$ & $\begin{array}{l}32 \\
29 \\
60\end{array}$ & $\begin{array}{l}26,5 \\
24,0 \\
49,5\end{array}$ & $\begin{array}{l}37 \\
36 \\
37\end{array}$ & $\begin{array}{l}33,6 \\
32,7 \\
33,6\end{array}$ & $\begin{array}{l}69 \\
65 \\
97\end{array}$ & $\begin{array}{l}29,9 \\
28,1 \\
42,0\end{array}$ \\
\hline Educação* & & & & & & 42,0 \\
\hline $\begin{array}{l}\text { Alta } \\
\text { Baixa }\end{array}$ & $\begin{array}{l}73 \\
48\end{array}$ & $\begin{array}{l}60,3 \\
39,7\end{array}$ & $\begin{array}{l}69 \\
41\end{array}$ & $\begin{array}{l}62,7 \\
37,3\end{array}$ & $\begin{array}{c}142 \\
89\end{array}$ & $\begin{array}{l}61,5 \\
38,5\end{array}$ \\
\hline $\begin{array}{l}\text { Quatificação } \\
\text { Profissional"* }\end{array}$ & & & & & & \\
\hline $\begin{array}{l}\text { Alta } \\
\text { Baixa }\end{array}$ & $\begin{array}{l}77 \\
44\end{array}$ & $\begin{array}{l}63,6 \\
36,4\end{array}$ & $\begin{array}{l}74 \\
36\end{array}$ & $\begin{array}{l}67,3 \\
32,7\end{array}$ & $\begin{array}{c}151 \\
80\end{array}$ & $\begin{array}{l}65,4 \\
34,6\end{array}$ \\
\hline
\end{tabular}

"Educaçāolalta = maior do que $01^{2} \mathrm{grau}$; baixa = igual ou menor do que o $1^{2}$ grau)

* Profissăo (alta = profissóes que requerem nivel de educaçăo superior, funçðes técnicas qualificadas: baixa=funçōes técnicas de média ou baixa qualificação, auxiliares ou atendentes
Tabela 5 - Médias de tensão arterial sistólica e diastólica de acordo com a disacusia ocupacional e história de exposiçăo ao ruido.

\begin{tabular}{|c|c|c|c|c|c|}
\hline \multirow{3}{*}{$\begin{array}{l}\text { Exposiçāo ao } \\
\text { Ruído Ocupacional }\end{array}$} & \multicolumn{5}{|c|}{ Tensāo arteria|* } \\
\hline & \multicolumn{2}{|c|}{ Sistólica } & \multicolumn{3}{|c|}{ Diastólica } \\
\hline & $N$ & Média & DP & Média & DP \\
\hline \multicolumn{6}{|l|}{ História referida } \\
\hline $\begin{array}{l}\text { Com } \\
\text { Sem }\end{array}$ & $\begin{array}{l}212 \\
64\end{array}$ & $\begin{array}{l}128,9 \\
124,8\end{array}$ & $\begin{array}{l}20,6 \\
21,0\end{array}$ & $\begin{array}{l}84,1 \\
82,7\end{array}$ & $\begin{array}{l}11,4 \\
15,1\end{array}$ \\
\hline \multicolumn{6}{|l|}{ Disacusia ocupacional } \\
\hline $\begin{array}{l}\text { Com } \\
\text { Sem }\end{array}$ & $\begin{array}{l}121 \\
110\end{array}$ & $\begin{array}{l}130,0 \\
126,0\end{array}$ & $\begin{array}{l}24,0 \\
18,5\end{array}$ & $\begin{array}{l}84,3 \\
83,1\end{array}$ & $\begin{array}{l}10,9 \\
13,2\end{array}$ \\
\hline
\end{tabular}

- Nenhuma das diferenças é estatisticamente significante para um alfa de 0.05 (Teste t de Student) $\mathrm{DP}=$ Desvio-Padră

Tabela 6 - Distribuiç̧̄o da hipertensão arterial de acordo com a disacusia ocupacional e história de exposiçăo ao ruido.

\begin{tabular}{|c|c|c|c|}
\hline \multirow{2}{*}{$\begin{array}{l}\text { Exposiçăo ao } \\
\text { Ruído Ocupacional }\end{array}$} & \multicolumn{3}{|c|}{ Hipertensäo arteria! } \\
\hline & Com & Sem & Total \\
\hline \multicolumn{4}{|l|}{ História referida } \\
\hline $\begin{array}{l}\text { Com } \\
\text { Sem }\end{array}$ & $\begin{array}{l}43 \\
15\end{array}$ & $\begin{array}{l}169 \\
49\end{array}$ & $\begin{array}{l}212 \\
64\end{array}$ \\
\hline Total & $\begin{array}{l}58 \\
\text { RP } 95 \% \mid C\end{array}$ & $\begin{array}{l}218 \\
0,87\end{array}$ & $\begin{array}{c}276 \\
(0,51,1,46)\end{array}$ \\
\hline $\begin{array}{c}\text { Disacusia ocupacio } \\
\text { Com } \\
\text { Sem }\end{array}$ & $\begin{array}{l}25 \\
23\end{array}$ & $\begin{array}{l}96 \\
87\end{array}$ & $\begin{array}{l}121 \\
110 \\
\end{array}$ \\
\hline Total & $\begin{array}{l}48 \\
\text { RP } 95 \% 1 \mathrm{C}\end{array}$ & $\begin{array}{r}183 \\
1,00\end{array}$ & $\begin{array}{c}231 \\
(0,59,1,64)\end{array}$ \\
\hline
\end{tabular}

RP = Razăo de proporçōes; $I C$ = Intervalo de confiança

nóstico de disacusia ocupacional, semelhante à proporçâo estimada $(20,9 \%)$ para os que se classificaram como portadores deste diagnóstico. Ainda na Tabela 6 pode ser visto que não houve evidências, nesse estudo, de associação entre hipertensão arterial e história de exposição ao ruído ocupacional ( $\mathrm{RP}=0,87,95 \%$ IC $: 0,83,1,11)$ ou disacusia ocupacional (RP=1,01,95\% IC:0,60, 1,64).

Considerando-se a história de exposição ao ruído ocupacional, os resultados da análise estratificada, apresentados na Tabela 7, mostram que este é um achado relativamente estável através das diferentes categorias das covariáveis. Vale notar o achado sugestivo de modificação de efeito observado com a variável eđucação, que ocorreu em nível limítrofe para um alfa $=0,05$. Ou seja, verificou-se que há um aumento da magnitude da medida de associação entre historia de exposição ao ruído e hipertensão arterial quando os indivíduos têm no máximo o primeiro grau completo. Quando a variável de 
exposição é a disacusia ocupacional, esta modificação da magnitude da medida de associação entre hipertensão arterial e exposição ao ruído, de acordo com a educaçāo, torna-se estatisticamente significante (Tabela 8). Especificamente, a razāo de proporçōes correspondente aos indivíduos de menor nível educacional é 2,3 (95\% IC: $0,8,6,8$ ), diferente da estimativa de 0,7 ( $95 \%$ IC: $0,4,1,3$ ) encontrada entre aqueles que referiram nível de educação igual ou acima do primeiro grau, desde que estes valores de RP não se encontram incluídos na faixa de valores compreendidos nos intervalos de confiança das categorias que lhes sāo opostas. Os demais achados de distribuição da exposição ao ruído, conforme pode ser avaliado pela disacusia ocupacional, assemelham-se àqueles identificados na análise da história referida de exposição ocupacional ao ruído. Nota-se aumento da força da associação entre as pessoas do sexo feminino ( $R P=2,195 \%$ IC: $0,7,7,0$ ) quando comparada àquela observada entre os homens ( $R P=1,095 \%$ IC: $0,6,1,7)$, apesar dessa diferença nāo atingir níveis de significância estatística.

A análise logística nâo-condicional foi conduzida, separadamente, para ambas as variáveis de exposicăo: história de exposição ao ruído ocupacional referida pelo trabalhador e diagnóstico de nexo causal para disacusia ocupacional. Dados relativos à avaliação da presença de va-

Tabela 7 - Razós de proporçỏes para a associaçăo entre a história referlda de exposição ocupacional ao ruido e hipertensáo arterial de acordo com as covariáveis do estudo.

\begin{tabular}{|c|c|c|c|}
\hline Covariáveis & $N=276$ & $\begin{array}{l}\text { Razáo de } \\
\text { proporçōes }\end{array}$ & $\begin{array}{l}\text { Intervalo de } \\
\text { confiança } 95 \%\end{array}$ \\
\hline $\begin{array}{l}\text { Medida bruta } \\
\text { Sexo }\end{array}$ & 276 & 0,9 & $(0,5,1,5)$ \\
\hline $\begin{array}{l}\text { Masc. } \\
\text { Fem. } \\
\text { ldade }\end{array}$ & $\begin{array}{l}243 \\
33\end{array}$ & $\begin{array}{l}0,9 \\
0,9\end{array}$ & $\begin{array}{l}(0,5,1,7) \\
(0,2,3,6)\end{array}$ \\
\hline $\begin{array}{c}19-33 \\
34-45 \\
45-64 \\
\text { Qualificaçăo } \\
\text { profissional* }^{*}\end{array}$ & $\begin{array}{l}81 \\
86 \\
109\end{array}$ & $\begin{array}{l}0,9 \\
1,3 \\
0,6\end{array}$ & $\begin{array}{l}(0,3,3,3) \\
(0,4,4,1) \\
(0,3,1,0)\end{array}$ \\
\hline $\begin{array}{c}\text { Alta } \\
\text { Baixa } \\
\text { Educaçåa }\end{array}$ & $\begin{array}{l}179 \\
97\end{array}$ & $\begin{array}{l}0,7 \\
1,3\end{array}$ & $\begin{array}{l}(0,4,1,3) \\
(0,4,4,3)\end{array}$ \\
\hline $\begin{array}{l}\text { Alta } \\
\text { Baixa }\end{array}$ & $\begin{array}{l}164 \\
112\end{array}$ & $\begin{array}{l}0,6 \\
1,8\end{array}$ & $\begin{array}{l}(0,4,1,1) \\
(0,6,5,7)\end{array}$ \\
\hline
\end{tabular}

* Profissão (alta = profissōes que requrem nivel de educaçào superior, funçðes técnicas qualificadas; baixa funçōes técnicas de média ou baixa qualificaçåo, auxiliares ou atendentes)

** Educaçáo (alta - major do que o $1^{2}$ grau; baixa = igual ou menor do que $01^{\circ}$ grau riáveis modificadoras de efeito, sob pressupostos multiplicativos, são mostrados na Tabela $9 \mathrm{e}$ 10. Consistentemente com o observado na análise tabuiar, verificou-se que o nível educacional causava modificação de efeito da medida de associaçāo mostrados na Tabela 9 e 10 quando a variável de exposição era a disacusia ocupacional: razão de verossimilhança de 10,16

Tabela 8 - Razóes de proporçðes para a associaçáo entre disacusia ocupacional $\theta$ hipertenșa arterial de acordo com as covariáveis do estudo.

\begin{tabular}{|c|c|c|c|}
\hline Covariáveis & $N=231$ & $\begin{array}{l}\text { Razóes de } \\
\text { proporçōes }\end{array}$ & $\begin{array}{l}\text { Intervalo de } \\
\text { Confiança } 95 \%\end{array}$ \\
\hline $\begin{array}{l}\text { Medida bruta } \\
\text { Sexo }\end{array}$ & 231 & 1,0 & $(0,6,1,6)$ \\
\hline $\begin{array}{l}\text { Masc. } \\
\text { Fem. } \\
\text { Idade }\end{array}$ & $\begin{array}{r}197 \\
34\end{array}$ & $\begin{array}{l}1,0 \\
2,1\end{array}$ & $\begin{array}{l}(0,6,1,7)^{*} \\
(0,7,6,9)\end{array}$ \\
\hline $\begin{array}{l}19-34 \\
35-41 \\
41-64\end{array}$ & $\begin{array}{l}69 \\
65 \\
97\end{array}$ & $\begin{array}{l}1,5 \\
1,0 \\
0,7\end{array}$ & $\begin{array}{l}(0,4,6,4) \\
(0,3,3,4) \\
(0,4,1,2)\end{array}$ \\
\hline $\begin{array}{l}\text { Qualificação* } \\
\text { profissional }\end{array}$ & & & \\
\hline $\begin{array}{l}\text { Ecucaçao } \\
\text { Alta } \\
\text { Baixa }\end{array}$ & $\begin{array}{r}142 \\
89\end{array}$ & $\begin{array}{l}0,7 \\
2,3\end{array}$ & $\begin{array}{l}(0,4,1,3)^{* *} \\
(0,8,6,8)\end{array}$ \\
\hline
\end{tabular}

- Nivel "bordeline" para nodificaçðes de efeito;

** Modificação de efeito, com diferenças estatisticamente significantes para um alfa 0,05 ;

*** Profissão (alta=profissóes que requeren nivel de educaçáo superior, funçóes técnicas qualificadas; baixa=funçðes técnicas de média ou baixa qualificação, auxiliares ou atendentes

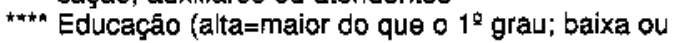
menor do que o $1^{\circ} \mathrm{grau}$ )

Tabela 9 - Resultados da análise de regressão logística na avallaçăo de confundimento para a associaçāo entre história de exposição ao ruído ocupacional ou disacusia. ocupacional e hipertensão arterial.

\begin{tabular}{|c|c|c|c|}
\hline $\begin{array}{l}\text { Variável independente } \\
\text { principal }\end{array}$ & $N$ & $\begin{array}{l}\text { ODDS } \\
\text { Ratio }\end{array}$ & $\begin{array}{l}\text { Intervalo de } \\
\text { contiança } 95 \%\end{array}$ \\
\hline \multicolumn{4}{|l|}{$\begin{array}{l}\text { I. História de expos|çăo } \\
\text { ao ruido ocupaclonal }\end{array}$} \\
\hline Modelo 2 - EXPP, SEX & 276 & 1,31 & $\begin{array}{l}(0,52,2,2) \\
\Delta=1,68 \\
(0,56,3,06) \\
\Delta=2,5\end{array}$ \\
\hline $\begin{array}{l}\text { II. Disacusia ocupacional } \\
\text { Modelo } 1 \text { - DIS }\end{array}$ & 231 & 1,02 & \multirow{2}{*}{$\begin{array}{l}(0,53,1,92) \\
\Delta=1,39 \\
(0,55,2,18) \\
\Delta=1,63\end{array}$} \\
\hline Modelo 2 - DIS, SEX & 231 & 1.10 & \\
\hline
\end{tabular}

$\Delta=0$ valor do limite superior menos o limite inferior

$\mathrm{DP}=$ Desvio-Padrão

EXPP = História de Exposição ao Ruldo Ocupacional

( $1=$ com, $0=\mathrm{sem}$ )

SEX $=$ Sexo $(1=$ masc. $0=$ fem. $)$

DIS = Disacusia Ocupacional $(1=\mathrm{com}, 0=5 \mathrm{em})$ 
Tabela 10 - Resultados da análise de regressāo logística para a avaliaçăo de variáveis modificadoras de efeito da associaçāo entre história de exposição ao ruído ocupacional, ou disacusia ocupacional e hipertensão arterial.

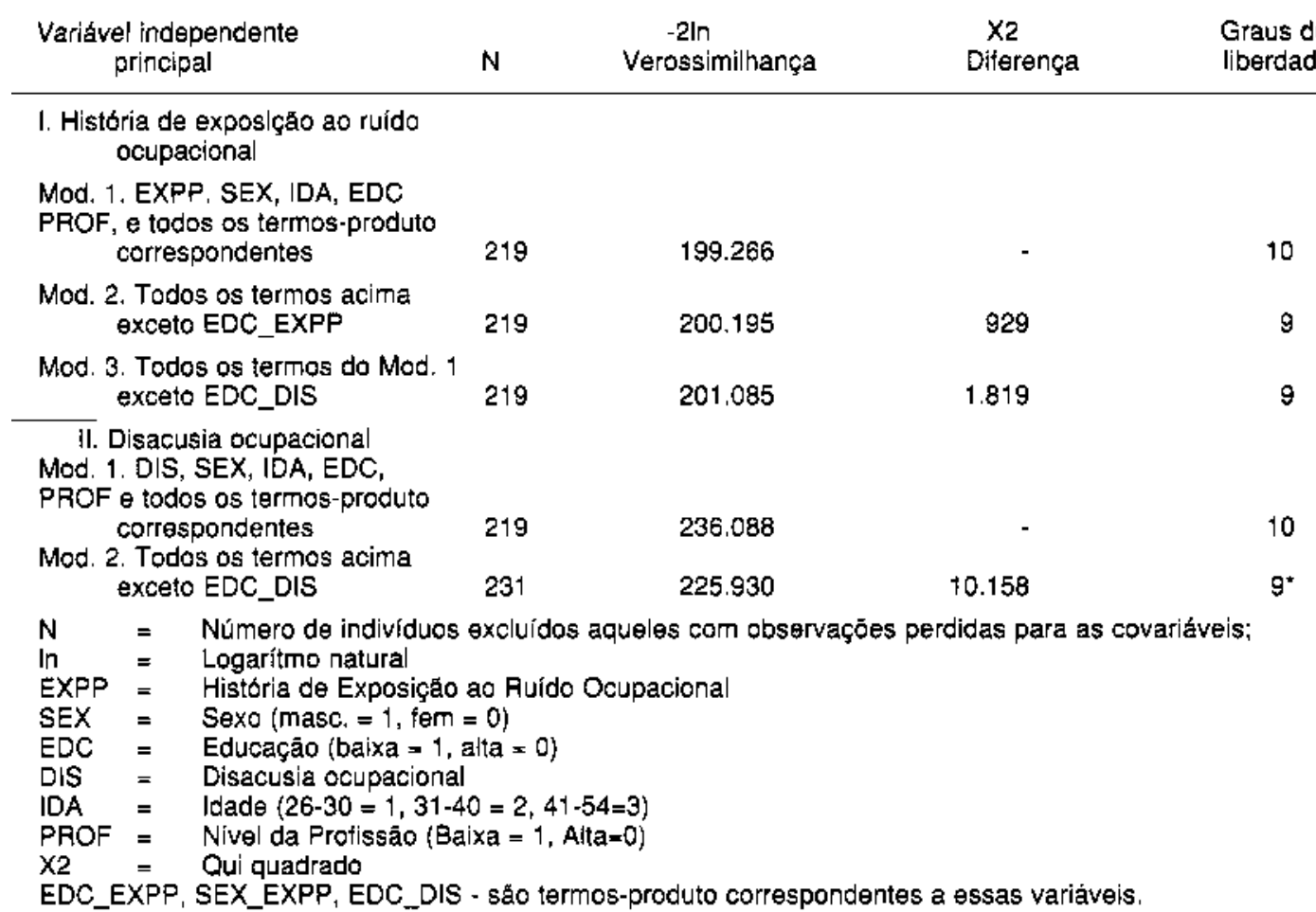

(1gl), estatisticamente significante $(p<0.05)$. Quanto à avaliação de confundimento, apenas a variável sexo foi examinada, posto que idade e qualificação profissional eram ambas relacionadas conceitualmente com a exposição, ou mais especificamente, com o nível de duração da exposição. Verificou-se que o ajustamento por sexo modificava substancialmente a associação entre história da exposição ao ruído ocupacional e hipertensão arterial, que passou de $\mathrm{OR}^{*}=1,09(95 \%$ IC: $0,52,2,20)$ para $1,31(95 \%$ IC: $0,56,3,06)$. Todavia, foi possível notar o concomitante incremento da imprecisäo da estimativa quando ajustada, tal qual pode ser verificada no aumento da amplitude do intervalo de confiança para a medida ajustada por este fator.

\section{Dlscussão}

A hipótese de que exposiçāo ao ruído ocupacional encontra-se positivamente associada com a hipertensão arterial não foi confirmada no presente estudo. Este resultado ocorreu consistentemente para ambas as variáveis indicativas de exposição ao ruído ocupacional, a história referida, como tam-

* OR - "odds ratio" ou razdo de produtos cruzados. bém com o diagnóstico de disacusia ocupacional, ou ainda com as médias tensionais, seja sistólica ou diastólica. Em reforço a esse achado, pode-se citar que não se observou nenhuma indicação de confirmação da hipótese em estudo, para nenhum dos estratos das covariáveis analisadas, apontando para uma relativa estabilidade dessa não associação, para ambas as medidas de exposição.

O presente estudo, de caráter exploratório, realizado com dados secundários provenientes de um serviço público de saúde ocupacional, as conclusões devem ser tomadas com precaução. Vejase, por exemplo, o elevado número de prontuários que nāo apresentaram dados referentes às variáveis principais, o que pode ter comprometido a distribuiçāo de co-ocorrência da exposição e do efeito, que não pôde ser avaliado no presente estudo. Embora a análise da distribuição das informaçōes perdidas năo tenha evidenciado tendências expressivas, este é um achado apenas sugestivo da não existência de viezes, porquanto a presença, magnitude e direção de tendenciosidades por perdas na população do estudo podem apenas ser definidas através da análise do padrão de ocorrência dessas perdas em relação à co-ocorrência da exposição e efeito avaliadas simultaneamente" ${ }^{\text {". }}$ Todavia, a redução do tamanho da amostra resultou em queda do poder do estudo: para uma dife- 
rença de proporções de 0,05 , assumindo-se um alfa de 0,05 (unicaudal) e um Beta de 0,20 , seria necessário estudar, aproximadamente 342 inđivíduos em cada um dos grupos de expostos e nāo expostos. Os tamanhos das amostras finais, com 276 e 231 indivíduos para a história de exposiçāo ao ruído e disacusia ocupacional, respectivamente, não atingem, portanto, o número mínimo suficiente para o teste da hipótese. Consequientemente, este estudo deve ser tomado apenas na perspectiva de uma análise exploratória. Outros fatores de seleção da populaçăo do estudo, como aqueles que determinam o fluxo de encaminhamentos e referência da demanda, que defínem uma diferença entre a populaçāo de base e do universo do estudo, sāo também possíveis fatores de distorção do efeito observado. Além desses problemas, não foi possível identificar ou quantificar possíveis viezes decorrentes da qualidade de informação dos prontuários, cujo preenchimento sem padronizaçāo pode ter trazido alterações nas co-variáveis. É conhecida a limitação do uso dessa fonte de dados em nosso meio, particularmente quando provenientes de serviços públicos de saúde ${ }^{15}$. No presente estudo, o dado referente ao tratamento antihipertensivo é crucial desde que os níveis tensionais se apresentassem "normalizados" na mensuração de indivíduos em tratamento. É possível que a ocorrência de tratamento não tenha sido registrada nos prontuários com o devido rigor.

Além de problemas devido à qualidade, não foi possível a obtençāo de dados relativos a fatores de risco conhecidos para a hipertensão arterial, que população de estudo poderiam ter um efeito confundidor potencial, tais como a obesidade ou o sobrepeso, o hábito de fumar, o consumo excessivo de bebidas alcóblicas ou o sedentarismo ${ }^{2.7 .13}$. Entre outras variáveis potencialmente confundíveis, que por definição seriam estranhas ao modelo térico construído para essa investigação, estariam outros fatores de risco para a hipertensão arterial, de natureza ocupacional ${ }^{7,8,10.17}$, que também não puderam ser contemplados na análise. Exemplo disso é a contaminação pelo chumbos que foi notada entre trabalhadores que compoem a demanda daquela unidade ambulatorial. Assim, o grupo de referência do presente estudo, trabalhadores com outros problemas de saúde, poderiam estar acometidos por enfermidades determinadas por exposiçôes ocupacionais, na qual a hipertensão é freqüentemente referida como efeito. Ou seja, se existe uma presumível elevaçăo da frequiência dessa enfermidade entre nāo expostos, o denominador da razāo de proporçōes estará artificialmente elevado, reduzindo-se, em consequiência, a magnitude da associaçăo, numa clara evidência de viés em torno da hipótese nula, ou seja, đe não associação. Estudos transversais são limitados para a identificação de evidências causais, por não permitirem, em sua maioria, estimativas do risco ou a determinaçāo da antecedência temporal entre exposiçāo e efeito. Na presente investigação, a falta de medidas apropriadas da exposiçăo impossibilitou a verificação de tendências dose-resposta, que em estudos transversais podem ser indicativas đe uma associação causal ${ }^{1}$.

Apesar das limitações da base populacional e fonte de dados, identificaram-se possíveis modificadores de efeito. Ou seja, observou-se excesso de casos de hipertensão arterial entre pessoas expostas ao ruído quando estas pertenciam ao sexo feminino, o que pode ser evidência de uma maior vulnerabilidade ao estresse das mulheres em face à este tipo de exposição. Observou-se a ocorrência de modificação de efeito, especificamente, um incremento da magnitude da medida de associaçāo quando os trabalhadores referiam um nivel educacional de no máximo o primeiro grau completo. Este achado foi verificado tanto na análise tabular como na multivariada, para pressupostos multiplicativos. A explicação para este resultado pode ser buscada na distribuiçăo social da exposicão ao ruído entre os trabalhadores. Ou seja, se esta exposição ao ruído ocorre, preferencialmente, en maior intensidade ou duração entre os indivíduos de menor nível educacional, isto levaria a que esta variável funcionasse como uma indicação de nível de exposição, determinando, portanto, o aumento da associação entre hipertensão e ruído neste grupo de educação. Como não houve a possibilidade de exploração analítica do gradiente biológico, ou dose-resposta, devido ao nível de agregaçāo da variável educaçāo nos prontuários, esta é apenas uma vertente interpretativa a ser avaliada em maior profundidade. Este achado não foi relatado em outros estudos, embora Zhao e col..$^{24}$ analisassem os seus dados nessa perspectiva. Pode-se concluir que embora nāo houvesse evidências de uma associação entre exposição ao rứdo e hipertensão arterial, observou-se uma modificaçāo de efeito, em nivel estatisticamente significante, $(a l f a=0,05)$ dessa associação com educação inferior ou igual ao primeiro grau e em nível "bordeline" com o sexo feminino que poderāo ser tomadas como objeto de estudos posteriores.

Estudos com resultados negativos - hipóteses sob investigaçāo nāo confirmadas - são frequiente- 
mente descartados para publicação, seja pelos seus próprios autores ou pelos comitês de periódicos. Pensa-se, todavia, que estes são dados também relevantes para apresentação à comunidade científica, uma vez que compõem uma parte do conhecimento produzido sob certas circunstâncias metodológicas. Este estudo exemplifica as limitações encontradas por muitos pesquisadores na conduçăo de pesquisa com dados secundários provenientes de serviços públicos de saúde, e pode vir a subsidiar decisões dirigidas à melhoria do registro de informaçōes, ainda que sejam simples medidas de rotina, como a tomada de pressão arterial. A escassez de estudos epidemiológicos, na área da saúde do trabalhador em nosso País, traduz as dificuldades por que passam aqueles que se defrontam com a fragilidade dos sistemas de informaçāo e do precário acesso às po-

\section{Referências Bibliográficas}

1. FLANDERS, D. et al. Assessing the direction of causality in cross-sectional studies. Am. J. Epidemiol., 135: 926-35, 1992.

2. FRASER, G. Preventive cardiology. New York. Oxford Univ. Press, 1986.

3. GLASS, D.C. \& SINGER, J.E, Urban stress - experiments on noise and social stressors. New York. Academic Press, 1972.

4. GREENIAND, S. \& ROBINS, J.M. Identifiability, exchangeability, and epidemiological confounding. Int. J. Epidemiol., 15: 413-9, 1986.

5. HERTZ-PICCIOTO, I. \& CROFT, J. A review of the relationship between blood lead and blood pressure. Chapel Hill. University of North Carolina, 1993.

6. HOSMER, D. \& LEMESHOW, S. Applied logistic regression. New York. John Wiley \& Sons, 1989. (Wiley Series in Probability and Mathematical Statistics).

7. JAMES, S, et al. John Henryism and blood pressure differences among black men. II The role of occupational stressors. J. Bebav. Med., 7: 259-75, 1984.

8. JENKINS, D.C. Epidemiology of cardiovascular diseases. J. Consul. Clin. Psycbol, 56: 324-32, 1988.

9. JONSSON, A. \& HANSON, L. Prolonged exposure to a stressful stimulus (noise) as a cause of raised blood-pressure in man. Lancet, 1: 86-7, 1977.

10. KARASEK, R. Control in the workplace and its health-related aspects. In: Sauter, S. L. ed, et al. Job control and worker's bealtb. New York. John Wiley \& Sons, 1989.

11. KLEINBAUM, D.G. et al. Epidemiologic researcb. principles and quantitative metbods. Wadsworth, Belmont, 1982.

12. LALRENTI, R. Epidemiologia das doenças cardiovasculares no Brasil, Arg. Bras. Cardiol, 38: 243-8, 1982.

13. LESSA, I. Estudos brasileiros sobre a epidemio- pulações de trabalhadores nos seus locais de trabalho, um problema já superado em algunas países, onde a preocupação com a identificação e o controle dos riscos ocupacionais é compartilhada pela sociedade como um todo.

\section{Agradecimentos}

Aos alunos das turmas TP02 e TP03 da disciplina "Epidemiologia" do curso de Medicina da Universidade Federal da Bahia, de 1993/primeiro semestre, e à equipe do Centro de Estudos da Saúde do Trabalhador que colaboraram na coleta de dados, como também aos colegas do Instituto de Saúde Coletiva e Departamento de Medicina Preventiva da UFBA, pelas sugestões.

logia da hipertensào arterial: anălise crítica dos estudos brasileiros. Inf. Epidemiol. SUS, 3(2): 59-75, 1993a.

14. LESSA, I. Epidemiologia da assistência médica e impacto economico e social das doenças cardiovasculares e do diabetes mellitus. Inf. Epidemiol. SUS, 4(2): 5-18, $1993 \mathrm{~b}$.

15. MACEDO et al. Assistência médica e mortalidade em Salvador-Bahia. Rev, Baiana Saude Püblica, 16: 7-14, 1989.

16. MALCHAIRE, J.B. \& MULLIER, M. Occupational exposure to noise and hypertension: a retrospective study. Ann. Occup. Hyg., 22: 63-73, 1979.

17. MARMOT, M. \& THEORELL, T. Social class and cardiovascular disease: the contribution of work. In: Johnson, J.V. \& Johansson, G. The psychosocial work environment; work organization, democratization and bealth - essays in memory of Bertil Gardell. Baywood Publishing Company Inc., New York, 1991. p.33-60.

18. ORGANIZACION MUNDIAL DE LA SALLD. Hipertension y cardiopatia coronária: classsificación y criterios para estudios epidemiologicos. Ginebra, 1961. Report. Geneva, 1962. (OMS - Série de Informes Técnicos, $n^{2} 168$ ).

19. PARVIZPOOR, D. Noise exposure and prevalence of high blood pressure among weavers in Iran. J. Occup. Med., 11(18) Nov., 1976.

20. PENA, P.G.L. Surdez profissional na Bahia: a historia social de uma doença do trabalho. [Dissertação de Mestrado, Salvador, 1990. Faculdade de Medicina da, Lniversidade Federal da Bahia].

21. SAS INSTITUTE, User's guide: Release 6.04 Edition. Cary, 1989.

22. TAKALA, J. Noise and blood pressure. Lancet, 2: 974-5, 1977

23. VERBEECK, J.H.A.M. et al. Non-auditory effects of noise in industry, Arch. Occup. Environ. Health, 59: 51-4, 1987.

24. $\mathrm{ZHAO}, \mathrm{Y}$. Dose response relationship for noiseinduced hypertension. Br. J. Ind. Med., 48: 179-84, 1991 


\begin{abstract}
The hypothesis that occupetional noise exposure is positively associated with hypertension was examined in a crosssectional study carried out on a group of patients who were enrolled at the Occupational Health Unit of the Unified Health System, situated in Salvador city, the capital of Bahia state, Brazil. Data were obtained from 276 medical records, corresponding to all patients newly registered during the first six months of 1992. Data on noise exposure come from both reported occupational exposure history and clinical diagnosis of occupational noise-induced hearing loss. Hypertension diagnosis complies with World Health Organization criteria, as well as with the history of antihypertensive treatment. Stratified analysis and unconditional logistic regression modeling show results that do not support the study hypothesis: there are no differences between systolic or diastolic blood pressure or between proportion of hepertension for exposed and non exposed groups. However, statiscally significant (alpha $=0.05$ ) increment of the effect measured was reported among workers who reported low educational level (below elementary). This could be another evidence of socially related inequalities underlying exposure distribution among workers at the workplace, which should be addressed, at greater depth, in future studies.
\end{abstract}

Hypertension, epidemiology. Noise, occupational. Hearing loss, noise induced, epidemiology. 